\title{
SEMICIRCLE LAW AND FREENESS FOR RANDOM MATRICES WITH SYMMETRIES OR CORRELATIONS
}

\author{
Jeffrey H. Schenker and Hermann Schulz-Baldes
}

\begin{abstract}
For a class of random matrix ensembles with correlated matrix elements, it is shown that the density of states is given by the Wigner semi-circle law. This is applied to effective Hamiltonians related to the Anderson model in dimensions greater than or equal to two.
\end{abstract}

\section{The result}

It is a classical theorem due to Wigner [Wig] that the density of states of a growing sequence of real symmetric matrices with independent entries converges in distribution to the semi-circle law. More precisely, this means the following: Consider an $n \times n$ random matrix $X_{n}=\left(\frac{1}{\sqrt{n}} a_{n}(p, q)\right)_{1 \leq p, q \leq n}$ where, apart from the symmetry condition, the entries $a_{n}(p, q)$ are independent centered random variables with unit variance (and a growth condition on their moments). Then the expectation value of the moment of $X_{n}$ of order $k \geq 0$ satisfies

$$
\lim _{n \rightarrow \infty} \mathbf{E} \frac{1}{n} \operatorname{Tr}_{n}\left(X_{n}^{k}\right)=\left\{\begin{array}{cc}
C_{\frac{k}{2}}=\frac{k !}{\frac{k}{2} !\left(\frac{k}{2}+1\right) !} & k \text { even }, \\
0 & k \text { odd } .
\end{array}\right.
$$

The coefficients $C_{k}$ are called the Catalan numbers, and the terms on the r.h.s. of (1) are precisely the moments of the semi-circle law, namely the absolutely continuous measure supported by the interval $[-2,2]$ with density $\frac{1}{2 \pi} \sqrt{4-x^{2}}$.

In this note we address the question: How many independent matrix elements are needed such that the above asymptotic behavior still holds? In other words, how many correlations may the matrix elements have? This is similar to an extension of the classical central limit theorem to sums of random variables with decaying correlations. We consider here a particular class of such correlations, including cases with supplementary symmetries of the matrix elements (apart from $\left.a_{n}(p, q)=a_{n}(q, p)\right)$. This is motivated by applications, in particular a simplified effective Anderson model. For the two-dimensional effective Hamiltonian, the so-called "flip-matrix" model introduced in [Poi], it was shown in $[\mathrm{BMR}]$ that the density of states is semi-circular using supersymmetric functional integrals. Bellissard has conjectured [Bel] that this result also holds for the effective Hamiltonians in higher dimensions. As a consequence of our result

Received by the editors January 31, 2005. 
we obtain a proof of this conjecture (see Section 2 for details). In Section 3, we indicate that known results on asymptotic freeness [Voi, Spe] extend to the present ensembles. This allows to calculate higher correlation functions.

The precise set-up we consider is as follows. For each $n \in \mathbb{N}$, suppose given an equivalence relation $\sim_{n}$ on pairs $P=(p, q)$ of indices in $\{1, \ldots, n\}^{\times 2}$. The entries of the matrix $X_{n}=\left(\frac{1}{\sqrt{n}} a_{n}(p, q)\right)_{1 \leq p, q \leq n}$ are complex random variables, with $a_{n}\left(p_{1}, q_{1}\right), \ldots, a_{n}\left(p_{j}, q_{j}\right)$ independent whenever $\left(p_{1}, q_{1}\right), \ldots,\left(p_{j}, q_{j}\right)$ belong to $j$ distinct equivalence classes of the relation $\sim_{n}$. Just as above, the entries are supposed to be centered, to have unit variance, and to obey a moment condition, namely

$$
m_{k}:=\sup _{n} \max _{p, q=1, \ldots, n} \mathbf{E}\left(\left|a_{n}(p, q)\right|^{k}\right)<\infty,
$$

for all $k \in \mathbb{N}$. For equivalent pairs $(p, q) \sim_{n}\left(p^{\prime}, q^{\prime}\right)$, the relation between $a_{n}(p, q)$ and $a_{n}\left(p^{\prime}, q^{\prime}\right)$ is not specified, and these variables may be correlated. For instance, we might have $a_{n}(p, q)=a_{n}\left(p^{\prime}, q^{\prime}\right)$, describing an auxiliary symmetry as in [Poi, BMR, Bel], but this is not necessary. However, the following conditions are imposed on the equivalence relation:

$$
\begin{aligned}
& \max _{p} \#\left\{\left(q, p^{\prime}, q^{\prime}\right) \in\{1, \ldots, n\}^{\times 3} \mid(p, q) \sim_{n}\left(p^{\prime}, q^{\prime}\right)\right\}=o\left(n^{2}\right) \\
& \max _{p, q, p^{\prime}} \#\left\{q^{\prime} \in\{1, \ldots, n\} \mid(p, q) \sim_{n}\left(p^{\prime}, q^{\prime}\right)\right\} \leq B
\end{aligned}
$$

for some constant $B<\infty$, and

$$
\#\left\{\left(p, q, p^{\prime}\right) \in\{1, \ldots, n\}^{\times 3} \mid(p, q) \sim_{n}\left(q, p^{\prime}\right) \& p \neq p^{\prime}\right\}=o\left(n^{2}\right) .
$$

Finally, we require that

$$
a_{n}(p, q)=\overline{a_{n}(q, p)},
$$

so that $X_{n}$ is Hermitian. Thus, $(p, q) \sim_{n}(q, p)$ for every $p, q$, which is consistent with (C1) to (C3).

Theorem 1. The density of states of an ensemble of Hermitian random matrices $X_{n}$ obeying the moment bound (2) and associated to equivalence relations $\sim_{n}$ satisfying $(\mathrm{C} 1),(\mathrm{C} 2)$ and $(\mathrm{C} 3)$ is a semicircle law, that is, (1) holds.

Remark: The first condition (C1) means that the size of the equivalence classes, i.e., the number of entries correlated with a given entry of the matrix, cannot grow too fast. The second condition (C2) is more technical, but plays a key role in the proof. It could be relaxed, replacing $B$ by $O\left(n^{\varepsilon}\right)$ for every $\varepsilon>0$, provided (C1) were strengthened, replacing $o\left(n^{2}\right)$ by $o\left(n^{2-\varepsilon}\right)$ for all $\varepsilon>0$. The third condition (C3) ensures, among other things, that there are not too many dependent rows, which could lead to many eigenvectors with eigenvalue zero. (An example illustrating this phenomenon is described below.) 
Our proof of this theorem is an extension of Wigner's original proof for the fully independent case [Wig, Spe, HP], the difference being that we use an equivalence relation on the pairs of indices rather than on the indices themselves (which may make Wigner's proof even more transparent to some readers).

Proof of Theorem 1. To begin, let us call a sequence $\left(P_{1}, \ldots, P_{k}\right)$ of pairs "consistent" if neighboring pairs $P_{l}=\left(p_{l}, q_{l}\right)$ and $P_{l+1}=\left(p_{l+1}, q_{l+1}\right)$ satisfy $q_{l}=p_{l+1}$, where $l=1, \ldots, k$ and $k+1$ is cyclically identified with 1 . Then, for any given $k \in \mathbb{N}$, we have

$$
\mathbf{E} \frac{1}{n} \operatorname{Tr}_{n}\left(X_{n}^{k}\right)=\frac{1}{n^{1+\frac{k}{2}}} \sum_{P_{1}, \ldots, P_{k}=(1,1)}^{(n, n)} \mathbf{E}\left(a_{n}\left(P_{1}\right) \ldots a_{n}\left(P_{k}\right)\right)
$$

where the sum runs over consistent sequences only. The terms of this sum are uniformly bounded as $n \rightarrow \infty$ :

$$
\left|\mathbf{E}\left(a_{n}\left(P_{1}\right) \ldots a_{n}\left(P_{k}\right)\right)\right| \leq m_{k},
$$

as follows from (2) and the Hölder inequality.

To group similar terms, let us associate a partition $\pi$ of $\{1, \ldots, k\}$ to each sequence $\left(P_{1}, \ldots, P_{k}\right)$ by means of

$$
l \sim_{\pi} m \quad \Longleftrightarrow \quad P_{l} \sim_{n} P_{m}
$$

and refer to $\left(P_{1}, \ldots, P_{k}\right)$ as a " $\pi$ consistent sequence". Introducing the notation $S_{n}(\pi)$ for the set of $\pi$ consistent sequences with indices in $\{1, \ldots, n\}$, equation (4) becomes

$$
\mathbf{E} \frac{1}{n} \operatorname{Tr}_{n}\left(X_{n}^{k}\right)=\frac{1}{n^{1+\frac{k}{2}}} \sum_{\pi \in \mathcal{P}(k)} \sum_{\left(P_{1}, \ldots, P_{k}\right) \in S_{n}(\pi)} \mathbf{E}\left(a_{n}\left(P_{1}\right) \cdots a_{n}\left(P_{k}\right)\right)
$$

where $\mathcal{P}(k)$ denotes the set of all partitions of $\{1, \ldots, k\}$.

Let $\# \pi$ be the number of blocks of the partition $\pi$. If $\# \pi>\frac{k}{2}$, then there has to be one singleton block, namely a block with only one element. Thus for any $\pi$ consistent sequence the corresponding entry in $a_{n}\left(P_{1}\right) \cdots a_{n}\left(P_{k}\right)$ appears only once. As this variable is centered and independent of all others appearing, $\mathbf{E}\left(a_{n}\left(P_{1}\right) \cdots a_{n}\left(P_{k}\right)\right)=0$. Therefore the sum in (5) can be restricted to partitions with less than or equal to $\frac{k}{2}$ blocks.

Next we argue that partitions with $r=\# \pi<\frac{k}{2}$ give vanishing contribution in the limit $n \rightarrow \infty$. Since the corresponding term in the sum over $\mathcal{P}(k)$ in $(5)$ is bounded by $m_{k} \times \# S_{n}(\pi)$, it suffices to derive an upper bound on the number of $\pi$ consistent sequences. Let us begin by choosing the pair $P_{1}$. There are $n^{2}$ choices to be made, corresponding to $n$ choices for each of the indices. Now, the first index of $P_{2}$ is fixed due to consistency. If $2 \sim_{\pi} 1$, then the second index can take at most $B$ values due to condition (C2), otherwise it is unconstrained, and can take at most $n$ values. Similarly, once we get to $P_{l}, l<k$, we have either at most $B$ or at most $n$ possible choices for the second index, depending 
on whether $l \sim_{\pi} j$ for one of $j=1, \ldots, l-1$. For the last pair $P_{k}$ there is no freedom, due to consistency. This shows

$$
\# S_{n}(\pi) \leq n^{2} n^{r-1} B^{k-r} .
$$

Since $r<\frac{k}{2}$, this contribution is negligible compared to the prefactor $1 / n^{1+k / 2}$ in (5) in the limit $n \rightarrow \infty$. In particular, this implies that the limit in (1) vanishes if $k$ is odd.

For even $k$, we may now focus on the contributions coming from partitions with exactly $k / 2$ blocks. Moreover, if the partition has a singleton the contribution vanishes as described above. Hence the partition has to be a pair partition, i.e., each block has exactly two elements. (Note that the "pair" in "pair partition" has nothing to do with the fact that each point $l$ is associated to a pair $P_{l}$ of indices.)

Let $\mathcal{P} \mathcal{P}(k)$ denote the pair partitions of $k$. To prove Theorem 1 , we must control somewhat more carefully the asymptotics in $n$ of $\# S_{n}(\pi)$ for a pair partition $\pi \in \mathcal{P} \mathcal{P}(k)$. This will be accomplished in the following four lemmas.

Lemma 1. Let $\pi \in \mathcal{P} \mathcal{P}(k)$ be a pair partition containing a pair of neighbors, that is $m \sim_{\pi} m+1$ for some $m$, and let $\pi^{\prime} \in \mathcal{P} \mathcal{P}(k-2)$ be the partition obtained by eliminating the corresponding pair (and relabeling $l \mapsto l-2$ for $m+2 \leq l \leq k$ ). Then

$$
\# S_{n}(\pi) \leq n \times \# S_{n}\left(\pi^{\prime}\right)+o\left(n^{\frac{k}{2}+1}\right) .
$$

Proof. Let us look at the situation close to $m$ and $m+1$. The indices are $\left(p_{m-1}, q_{m-1}\right),\left(p_{m}, q_{m}\right),\left(p_{m+1}, q_{m+1}\right)$ and $\left(p_{m+2}, q_{m+2}\right)$. By consistency, we have $q_{m}=p_{m+1}$. Now consider separately the two cases (i) $p_{m}=q_{m+1}$ and (ii) $p_{m} \neq q_{m+1}$.

In case (i), after two applications of the consistency condition, we get $q_{m-1}=$ $p_{m+2}$. Hence after eliminating the pairs $P_{m}$ and $P_{m+1}$, we have a $\pi^{\prime}$ consistent sequence. Therefore, in this case there are $n$ choices for $q_{m}=p_{m+1}$ and at most $\# S_{n}\left(\pi^{\prime}\right)$ choices for the remaining indices, giving the first term in (6).

In case (ii), by (C3) there are only $o\left(n^{2}\right)$ choices for the triple $p_{m}, q_{m}=$ $p_{m+1}, q_{m+1}$. Since there are $(k-2) / 2$ pairs remaining, there are no more than $n^{(k-2) / 2} B^{(k-2) / 2-1}$ choices for the remaining indices. To see this, start at $m+2$ and proceed through the remaining indices cyclically as in the argument to eliminate partitions with $\# \pi<k / 2$ above. Combining the two factors $o\left(n^{2}\right) \times$ $O\left(n^{(k-2) / 2}\right)$ gives the second term in $(6)$.

A partition $\pi$ is called crossing if there are positions $m_{1}<m_{2}<m_{3}<m_{4}$ such that $m_{1} \sim_{\pi} m_{3}$ and $m_{2} \sim_{\pi} m_{4}$, otherwise it is called non-crossing.

Lemma 2. Let $\pi \in \mathcal{P} \mathcal{P}(k)$ be a crossing pair partition, then

$$
\lim _{n \rightarrow \infty} \frac{\# S_{n}(\pi)}{n^{\frac{k}{2}+1}}=0
$$


Proof. The first step is to apply Lemma 1 as many times as possible, eliminating all nearest neighbor pairs in $\pi$ and the resulting reduced partitions. The end result is that

$$
\# S_{n}(\pi) \leq n^{r} \# S_{n}\left(\pi^{\prime}\right)+o\left(n^{\frac{k}{2}+1}\right),
$$

where $r$ is the number of pairs eliminated, and $\pi^{\prime} \in \mathcal{P} \mathcal{P}(k-2 r)$ is maximally crossing in the sense that it cannot be further reduced by the procedure of Lemma 1, i.e., for every $m$ we have $m \chi_{\pi^{\prime}} m+1$. Because $\pi$ is crossed, there are at least two pairs left, so $k-2 r \geq 4$. Consider a pair $m \sim_{\pi^{\prime}} m+\ell$ of $\pi^{\prime}$ with minimal $\ell$, understood in a cyclic sense on the $k-2 r$ positions of $\pi^{\prime}$. Then $\ell \geq 2$ and all points $j$ with $m<j<m+\ell$ form pairs with points outside $\{m, \ldots, m+\ell\}$, i.e., these pairs cross the pair $m \sim_{\pi^{\prime}} m+\ell$.

To estimate $\# S_{n}\left(\pi^{\prime}\right)$, let us first count the choices for indices in the interval $\{m, \ldots, m+\ell\}$, first choosing $P_{m}=\left(p_{m}, q_{m}\right), P_{m+\ell}=\left(p_{m+\ell}, q_{m+\ell}\right)$, and then successively $P_{m+1}=\left(p_{m+1}, q_{m+1}\right), P_{m+2}, \ldots$ There are $n$ choices for $p_{m}$ and then, by $(\mathrm{C} 1), o\left(n^{2}\right)$ choices for the three indices $q_{m}, p_{m+\ell}, q_{m+\ell}$. Now, $p_{m+1}$ is fixed by consistency, but $q_{m+1}$ is unconstrained aside from the requirement $P_{m+1} \chi_{n} P_{m}$, which we ignore to obtain an upper bound. The same holds for $P_{m+2}, \ldots, P_{m+\ell-2}$, however, for $P_{m+\ell-1}$ there is no freedom due to consistency. For the whole interval $\{m, \ldots, m+\ell\}$ this gives an upper bound $n \times o\left(n^{2}\right) \times$ $n^{\ell-2}=o\left(n^{\ell+1}\right)$ on the number of choices.

Finally, let us go through the remaining $k-2 r-\ell+1$ pairs of indices in increasing order (cyclically), starting with $P_{m+\ell+1}$. Consistency fixes the first index. If $m+\ell+1$ is paired under $\pi^{\prime}$ with one of the previously considered points, then (C2) fixes also the second index within a set of size no larger than $B$. If $m+\ell+1$ does not form a pair with any previous point, then there are at most $n$ choices for the second index. As we go through the remaining $k-2 r-\ell-1$ pairs, the latter case occurs exactly $\frac{k-2 r}{2}-\ell$ times. We deduce that

$$
\# S_{n}\left(\pi^{\prime}\right) \leq n^{r} \times o\left(n^{\ell+1}\right) \times n^{\frac{k}{2}-r-\ell} B^{\frac{k}{2}-r-1}+o\left(n^{\frac{k}{2}+1}\right)=o\left(n^{\frac{k}{2}+1}\right) .
$$

Thus we may restrict the sum in (5) to non-crossing pair partitions of $\{1, \ldots, k\}$, denoted by $\mathcal{N} \mathcal{P} \mathcal{P}(k)$. For $\pi \in \mathcal{N} \mathcal{P} \mathcal{P}(k)$, let $P S_{n}(\pi)$ denote the subset of $S_{n}(\pi)$ made up of $\pi$ consistent sequences such that for any two sites $m, m^{\prime}$ paired by $\pi, m \sim_{\pi} m^{\prime}$, we have $P_{m^{\prime}}=\left(q_{m}, p_{m}\right)$, where $P_{m}=\left(p_{m}, q_{m}\right)$. Note that for $\left(P_{1}, \ldots, P_{k}\right) \in P S_{n}(\pi)$ we have

$$
\mathbf{E}\left(a_{n}\left(P_{1}\right) \cdots a_{n}\left(P_{k}\right)\right)=1,
$$

since $a_{n}\left(P_{m}\right)=\overline{a_{n}\left(P_{m^{\prime}}\right)}$ for equivalent sites $m \sim_{\pi} m^{\prime}$ and the $a_{n}(P)$ have unit variance. Furthermore, there are not too many sequences missed by $P S_{n}(\pi)$ :

Lemma 3. Let $\pi \in \mathcal{N} \mathcal{P} \mathcal{P}(k)$ be non-crossing. Then

$$
\lim _{n \rightarrow \infty} \frac{\# S_{n}(\pi)-\# P S_{n}(\pi)}{n^{\frac{k}{2}+1}}=0
$$


Proof. Let $N S_{n}(\pi)=S_{n}(\pi) \backslash P S_{n}(\pi)$. Repeating the proof of Lemma 2, we see that

$$
\# N S_{n}(\pi) \leq n \times \# N S_{n}\left(\pi^{\prime}\right)+o\left(n^{\frac{k}{2}+1}\right),
$$

where $\pi^{\prime}$ is a partition of $k-2$ obtained by removing a nearest neighbor pair from $\pi$. Referring to the notation of the proof of Lemma 1, the only new feature here is that in case (i), after eliminating the pair $P_{m} \sim_{n} P_{m+1}$ (and relabeling $m+2 \mapsto m$, etc.), we get a sequence in $N S_{n}\left(\pi^{\prime}\right)$. This follows because the eliminated pair was of the form $(p, q) \sim_{n}(q, p)$, so the "defect" is still present in the reduced sequence.

Iteration of the above gives

$$
N S_{n}(\pi) \leq n^{\frac{k}{2}-1} \# N S_{n}\left(\pi^{\prime \prime}\right)+o\left(n^{\frac{k}{2}+1}\right),
$$

where $\pi^{\prime \prime} \in \mathcal{P} \mathcal{P}(2)$ is a pair partition for which \#NS$S_{n}\left(\pi^{\prime \prime}\right)=o\left(n^{2}\right)$, by condition (C3).

After restricting to pair partitions in (5) and applying Lemmas 1 to 3 and equation (7), we conclude that

$$
\lim _{n \rightarrow \infty} \mathbf{E} \frac{1}{n} \operatorname{Tr}_{n}\left(X_{n}^{k}\right)=\lim _{n \rightarrow \infty} \frac{1}{n^{1+\frac{k}{2}}} \sum_{\pi \in \mathcal{N} \mathcal{P} \mathcal{P}(k)} \# P S_{n}(\pi),
$$

where, by the following lemma, the limit on the r.h.s. is in fact just the number of non-crossing pair partitions.

Lemma 4. If $\pi \in \mathcal{N} \mathcal{P} \mathcal{P}(k)$, then

$$
\lim _{n \rightarrow \infty} \frac{\# P S_{n}(\pi)}{n^{\frac{k}{2}+1}}=1
$$

Proof. We first note that $\frac{k}{2}-1$ applications of Lemma 1 imply

$$
\# S_{n}(\pi) \leq n^{\frac{k}{2}-1} \times \# S_{n}\left(\pi^{\prime}\right)+o\left(n^{\frac{k}{2}+1}\right),
$$

where $\pi^{\prime} \in \mathcal{P} \mathcal{P}(2)$ is a pair partition, for which $\# S_{n}\left(\pi^{\prime}\right)=n^{2}+o\left(n^{2}\right)$, by $(\mathrm{C} 3)$. Thus

$$
\# P S_{n}(\pi) \leq \# S_{n}(\pi) \leq n^{\frac{k}{2}+1}+o\left(n^{\frac{k}{2}+1}\right) .
$$

In order to prove a lower bound, let us count the number of choices for indices, proceeding from left to right. We first have exactly $n^{2}$ choices for $\left(p_{1}, q_{1}\right)$. This determines $p_{2}=q_{1}$ by consistency. If $1 \sim_{\pi} 2$ then $q_{2}=p_{1}$ is fixed since we require a $P S_{n}(\pi)$ sequence, otherwise there remain exactly $n-1$ choices for $q_{2}$ (since $q_{2}=p_{1}$ is forbidden because $1 \chi_{\pi} 2$ ). More generally, once we reach the $j^{\text {th }}$ pair of indices, $p_{j}=q_{j-1}$ is fixed by consistency, but $q_{j}$ is fixed if and only if $j$ is paired under $\pi$ with a number $i<j$. If $j$ is not paired with a prior number, there remain at least $n-(j-1) B \geq n-(k-1) B$ choices for $q_{j}$. Indeed this follows from $(\mathrm{C} 2)$ since $q_{j}$ is free up to the constraint that $\left(p_{j}, q_{j}\right) \chi_{n}\left(p_{i}, q_{i}\right)$ for $i<j$. We obtain in this way the lower bound

$$
\# P S_{n}(\pi) \geq n^{2}(n-(k-1) B)^{\frac{k}{2}-1} \geq(n-(k-1) B)^{\frac{k}{2}+1},
$$


since there are exactly $k / 2$ pairs in $\pi$, and thus $k / 2+1$ free indices.

Combining the two bounds (9) and (10) completes the proof.

This concludes the proof of Theorem 1, because the number of non-crossing partitions, $\# \mathcal{N} \mathcal{P} \mathcal{P}(k)$, is precisely equal to the Catalan number $C_{\frac{k}{2}}$ [Spe, HP].

\section{Examples}

2.1. Flip matrix model. For each $n$, let $\Phi_{n}:\{1, \ldots, n\} \rightarrow\{1, \ldots, n\}$ be the involution $\Phi_{n}(p)=n+1-p$, and take for the equivalence relation

$$
(p, q) \sim_{n}(q, p) \sim_{n}\left(\Phi_{n}(p), \Phi_{n}(q)\right) \sim_{n}\left(\Phi_{n}(q), \Phi_{n}(p)\right) .
$$

Clearly (C1) and (C2) are satisfied. For even $n$, (C3) holds because $(p, q) \sim_{n}$ $\left(q, p^{\prime}\right)$ if and only if $p=p^{\prime}$. For odd $n$, this is true except for pairs of the form $\left(p, \frac{n+1}{2}\right) \sim_{n}\left(\frac{n+1}{2}, \Phi_{n}(p)\right)$, since $\frac{n+1}{2}$ is a fixed point of $\Phi_{n}$. As there are only $n-1$ such pairs, (C3) holds. Thus, Theorem 1 applies and the density of states is a semi-circle law.

Restricting to even $n$ and taking for the matrix elements complex Gaussians, we obtain the flip matrix model of [Poi]. Theorem 1 in this special case reproduces a result derived in $[\mathrm{BMR}]$. We comment below on how this ensemble appears in connection with the Anderson model.

2.2. Condition (C3). The importance of condition (C3) is illustrated by the following example. Let the equivalence relation be induced by

$$
(p, q) \sim_{n}(q, p) \sim_{n}\left(\Phi_{n}(p), q\right) \sim_{n}\left(\Phi_{n}(q), \Phi_{n}(p)\right),
$$

where $\Phi_{n}$ is as above. Unless $p$ or $q$ are fixed points of $\Phi_{n}$, the class of $(p, q)$ contains 8 elements. Conditions (C1) and (C2) are clearly satisfied. However, (C3) is violated since $(p, q) \sim_{n}\left(q, \Phi_{n}(p)\right)$ for every $p, q$, giving at least $n \times(n-1)$ pairs $(p, q) \sim_{n}\left(q, p^{\prime}\right)$ with $p \neq p^{\prime}$. Consider, associated to this $\sim_{n}$, an ensemble of real symmetric matrices with $a_{n}(P)=a_{n}\left(P^{\prime}\right)$ when $P \sim_{n} P^{\prime}$. The matrix $X_{n}$ has a $[n / 2]$ dimensional null space (here $[a]$ denotes the integer part of $a \in \mathbb{R}$ ), consisting of vectors $\left(v_{1}, \ldots, v_{n}\right)$ with $v_{j}=-v_{n+1-j}$. As a result, the density of states for this ensemble has an atom at 0 . In fact, the density of states can be computed by noting that

$$
X_{n} \cong \frac{1}{\sqrt{2}}\left(\begin{array}{ll}
1 & 1 \\
1 & 1
\end{array}\right) \otimes Y_{n / 2} \cong\left(\begin{array}{cc}
\sqrt{2} & 0 \\
0 & 0
\end{array}\right) \otimes Y_{n / 2},
$$

where $\cong$ denotes unitary equivalence and $Y_{n}$ is an ensemble of matrices satisfying the conditions of Theorem 1. Thus the density of states is a superposition of an atom at 0 and a scaled semi-circle law:

$$
\frac{1}{2} \delta(x) \mathrm{d} x+\frac{1}{2} \frac{1}{2 \pi} \sqrt{4-2 x^{2}} \chi\left(x^{2} \leq 2\right) \sqrt{2} \mathrm{~d} x .
$$


2.3. Higher dimensional flip models. Let us first describe how these models appear as effective Hamiltonians for a finite size approximation of the Anderson model in the weak coupling limit, and then apply Theorem 1 to them.

The Anderson Hamiltonian $H=H_{0}+V$ on the $\ell^{2}$ space over a square lattice is the sum of a translation invariant part $H_{0}=H_{0}^{*}$ and a (small) random potential $V$. Consider such an operator in a finite volume

$$
\Lambda_{L}=\left\{-\frac{L}{2}+1, \ldots, \frac{L}{2}\right\}^{d},
$$

together with periodic boundary conditions. Then $V$ is a multiplication operator on $\ell^{2}\left(\Lambda_{L}\right)$ given by a random real-valued function $v$ on $\Lambda_{L}$. Furthermore the orthonormal basis $\phi_{p}(x)=\mathrm{e}^{i p \cdot x} / L^{d / 2}$, with quasimomentum $p$ in the (discrete) Brillouin zone $\Lambda_{L}^{*}=\frac{2 \pi}{L} \Lambda_{L}$, consists of eigenfunctions of $H_{0}$ with eigenvalues $\mathcal{E}(p)$, where $\mathcal{E}$ is some smooth periodic function on the torus $[-\pi, \pi)^{d}$ - the so-called symbol of $H_{0}$.

For small $V$, the spectral analysis of $H$ near a fixed energy $E$ involves primarily states $\phi_{p}$ with quasimomenta in the discrete Fermi surface

$$
\Lambda_{L}^{*}(E)=\left\{p \in \Lambda_{L}^{*}|| \mathcal{E}(p)-E \mid \leq \frac{\beta}{L}\right\},
$$

with $\beta>0$ a parameter giving its width. The restriction of the random potential to the corresponding subspace will be the higher dimensional flip model. It is hence the lowest order in a nearly degenerate perturbation theory for the Anderson Hamiltonian at scale $L$.

More precisely, the multiplication operator $\psi(x) \mapsto v(x) \psi(x)$ acts on the basis $\phi_{p}$ as follows:

$$
\phi_{p} \mapsto \sum_{q \in \Lambda_{L}^{*}} \widehat{v}(p-q) \phi_{q},
$$

where $\widehat{v}$ is the (finite sum) Fourier transform of $v$, i.e.,

$$
\widehat{v}(p)=\frac{1}{L^{d}} \sum_{x \in \Lambda_{L}} v(x) \mathrm{e}^{i p \cdot x},
$$

and $p-q$ is evaluated modulo $2 \pi$. Restriction to the Fermi surface gives thus the following convolution operator $A_{\omega}$ on $\ell^{2}\left(\Lambda_{L}^{*}(E)\right)$

$$
A_{\omega} \phi(p)=\sum_{q \in \Lambda_{L}^{*}(E)} \widehat{v}(p-q) \phi(q) .
$$

Hence we are led to introduce the random matrix

$$
Y_{n}(p, q)=\widehat{v}(p-q), \quad p, q \in \Lambda_{L}^{*}(E) .
$$

Here $n=\# \Lambda_{L}^{*}(E)=O\left(L^{d-1}\right)$.

If one takes $v(x)$ to be independent and identically distributed centered Gaussians, then the variables $\widehat{v}(p)$ are also centered Gaussians, which are mutually independent apart from the constraint $\overline{\widehat{v}(p)}=\widehat{v}(-p)$ which results because the $v(x)$ are real. In terms of the variance $\lambda^{2}=\mathbf{E}\left(v(x)^{2}\right)$, the variance of $\widehat{v}(p)$ is 
$\lambda^{2} / L^{d}$. The higher dimensional flip matrix model is the random matrix $Y_{n}$ defined in (12), with the index set given by (11), where random variables $\widehat{v}(p)$ have variance $\lambda^{2} / L^{d}$ and satisfy the constraint $\overline{\widehat{v}(p)}=\widehat{v}(-p)$, as well as the bounds

$$
\max _{p \in \Lambda_{E}^{*}} \mathbf{E}\left(|\widehat{v}(p)|^{k}\right) \leq M_{k} \lambda^{k} L^{-\frac{k d}{2}} .
$$

To simplify the discussion, we suppose that $\mathcal{E}(p)=p^{2} / 2$ in a neighborhood of $E>0$, so that $\Lambda_{L}^{*}(E)$ is the intersection of a spherical shell with the lattice $\Lambda_{L}^{*}$. This eases some arguments, but does not affect the end result provided the level sets $\left\{p \in \Lambda_{L}^{*} \mid \mathcal{E}(p)=E \pm \delta\right\}$, for small $\delta$, are sufficiently regular and have curvature bounded away from zero.

Remark: The flip matrices of Section 2.1 are a simplification of $Y_{n}$ in $d=2$ obtained via a course graining procedure. The shell $\Lambda_{L}^{*}(E)$ is divided into $m$ blocks, each subtending an angle of size $O\left(L^{-\frac{1}{2}}\right)$. Each block contains $O\left(L^{-\frac{1}{2}} \times\right.$ $\left.L^{-1} \times L^{2}\right)=O\left(L^{\frac{1}{2}}\right)$ points. The flip matrix $F_{m}\left(b, b^{\prime}\right)$ is indexed by pairs of blocks, with Gaussian $F_{m}\left(b, b^{\prime}\right)$ which are pairwise independent except that

$$
F_{m}\left(b, b^{\prime}\right)=\overline{F_{m}\left(b^{\prime}, b\right)}=F_{m}\left(-b^{\prime},-b\right)=\overline{F_{m}\left(-b,-b^{\prime}\right)} .
$$

The above coarse graining was introduced in [Poi, BMR] as a simplification to avoid the combinatorics required to deal with matrix elements $Y_{n}(p, q)$ with $p-q$ small. We do not need coarse graining here, because Theorem 1 applies directly to an appropriate rescaling of $Y_{n}$.

We now turn to the analysis of the higher dimensional flip model. The matrix elements $Y_{n}(p, q)$ and $Y_{n}\left(p^{\prime}, q^{\prime}\right)$ are statistically independent unless $p-q= \pm\left(p^{\prime}-\right.$ $q^{\prime}$ ), and thus we define $\sim_{n}$ accordingly:

$$
(p, q) \sim_{n}\left(p^{\prime}, q^{\prime}\right) \Longleftrightarrow p-q= \pm\left(p^{\prime}-q^{\prime}\right)
$$

Condition (C2) is clearly satisfied, but conditions $(\mathrm{C} 1)$ and $(\mathrm{C} 3)$ require proof.

Condition (C3) is a bit easier to verify. The relevant pairs $(p, q) \sim_{n}\left(q, p^{\prime}\right)$ with $p \neq p^{\prime}$ have to satisfy $p^{\prime}=2 q-p$. Fixing $q \in \Lambda_{L}^{*}(E)$, this implies that the conditions $p \in \Lambda_{L}^{*}(E)$ and $2 q-p \in \Lambda_{L}^{*}(E)$ have to hold simultaneously. The relevant set of $p$ 's is contained in the intersection of two spherical shells:

$$
\left\{\left|p^{2} / 2-E\right| \leq \frac{\beta}{L}\right\} \cap\left\{\left|(p-2 q)^{2} / 2-E\right| \leq \frac{\beta}{L}\right\} .
$$

As $|q|^{2} / 2=E$, these shells are barely touching and there are

$$
O\left(L^{-\frac{d+1}{2}} L^{d}\right)=O\left(L^{\frac{d-1}{2}}\right)=o\left(L^{d-1}\right)=o(n)
$$

points in the intersection of this set with $\Lambda_{L}^{*}(E)$, essentially because the $d$ dimensional volume of the set is $O\left(L^{-\frac{d+1}{2}}\right)$ and the lattice cell volume of $\Lambda_{L}^{*}$ is $(2 \pi)^{d} / L^{d}$. As this holds for all $n$ values of $q$, we conclude that (C3) holds.

For $(\mathrm{C} 1)$, we need to estimate, given a fixed value of $p$, the number of triples $q, p^{\prime}, q^{\prime} \in \Lambda_{L}^{*}(E)$ with $(p, q) \sim_{n}\left(p^{\prime}, q^{\prime}\right)$. First note that

$$
q^{\prime}=p^{\prime} \pm(p-q)
$$


so that $q^{\prime}$ takes 2 values once $q$ and $p^{\prime}$ are chosen. Furthermore

$$
\left|q^{\prime}\right|^{2}-\left|p^{\prime}\right|^{2}= \pm 2 p^{\prime} \cdot(p-q)+|p-q|^{2} \text {. }
$$

Because $p^{\prime}, q^{\prime} \in \Lambda_{L}^{*}(E)$, this implies

$$
\left|\frac{|p-q|}{2} \pm p^{\prime} \cdot \frac{(p-q)}{|p-q|}\right| \leq \frac{2 \beta}{L|p-q|} .
$$

In order to count the number of possible values for $q$ and $p^{\prime}$, we consider separately the cases of large and small $p-q$. If $|p-q| \geq L^{-\frac{1}{2}}$, then

$$
\left|\frac{|p-q|}{2\left|p^{\prime}\right|} \pm \cos \theta\right| \leq \frac{2 \beta}{\left|p^{\prime}\right| \sqrt{L}},
$$

where $\theta$ is the angle between $p^{\prime}$ and $(p-q)$. Because for all $t$

$$
|\{\theta \in[-\pi, \pi]|| \cos \theta-t \mid \leq \delta\}| \leq \sqrt{2 \delta}
$$

we conclude that the allowed set of $p^{\prime}$ is contained in a spherical cone which subtends an angle no larger than $O\left(L^{-\frac{1}{4}}\right)$. The intersection of this spherical cone with $\Lambda_{L}^{*}(E)$ contains $O\left(L^{-1-\frac{1}{4}(d-1)} \times L^{d}\right)=O\left(L^{\frac{3}{4}(d-1)}\right)$ points. As there are $O\left(L^{d-1}\right)$ points $q \in \Lambda_{L}^{*}(E)$ with $|p-q| \geq L^{-\frac{1}{2}}$, we end up with no more than $O\left(L^{\frac{7}{4}(d-1)}\right)$ triples in this case. However, in the other case there are only $O\left(L^{-1-\frac{1}{4}(d-1)} \times L^{d}\right)=O\left(L^{\frac{3}{4}(d-1)}\right)$ points $q \in \Lambda_{L}^{*}(E)$ with $|p-q| \leq L^{-\frac{1}{2}}$, and thus no more than $O\left(L^{\frac{3}{4}(d-1)} \times L^{d-1}\right)=O\left(L^{\frac{7}{4}(d-1)}\right)$ triples. Altogether we conclude that

$$
\#\left\{\left(q, p^{\prime}, q^{\prime}\right) \in \Lambda_{L}^{*}(E) \mid(p, q) \sim_{n}\left(p^{\prime}, q^{\prime}\right)\right\}=O\left(L^{\frac{7}{4}(d-1)}\right)=o\left(L^{2(d-1)}\right),
$$

and thus that $(\mathrm{C} 1)$ holds.

Therefore, Theorem 1 applies to $X_{n}=c_{n} Y_{n}$ with a scalar $c_{n}$ chosen so that the variance of the matrix elements of $X_{n}$ is $1 / n$, namely $c_{n}^{2}=\left(L^{d} / \lambda^{2}\right) \times n^{-1}=$ $O(L) / \lambda^{2}$. In the context of the Anderson model, it is natural [Poi, Bel] to take $L \approx \lambda^{-2}$, giving $n(\lambda)=\# \Lambda_{L}^{*}(E)=O\left(\lambda^{2-2 d}\right)$. Then $Y_{n(\lambda)}(p, q) \approx \lambda^{2} \times$ $X_{n(\lambda)}(p, q)$ where the $X_{n}$ are matrices which satisfy the hypotheses of Theorem 1 . It follows that the density of states of $Y_{n(\lambda)}$ is asymptotic as $\lambda \rightarrow 0$ to a scaled semi-circle law of width of order $\lambda^{2}$.

\section{Asymptotic freeness}

The semicircle law is the only stable law of Voiculescu's free probability theory for which all moments exist, playing a role there analogous to that of the Gaussian in classical probability. The above techniques also allow to extend known results on asymptotic freeness of random matrices [VDN, Voi, Spe, HP]. Suppose given a family $\left(X_{n}^{(i)}\right)_{i \in I}$ of random $n \times n$ matrices with some index set $I$. For different indices $i$, the entries of the matrices are supposed to be independent, and for each $i$, the family $\left(X_{n}^{(i)}\right)_{n \in \mathbb{N}}$ is specified by a possibly $i$-dependent equivalence relation satisfying conditions (C1), (C2) and (C3). Furthermore suppose given a second family $\left(D_{n}^{(j)}\right)_{j \in J}$ of diagonal real random matrices, the entries 
of which are also independent among themselves and with respect to the family $\left(X_{n}^{(i)}\right)_{i \in I}$. Each $\left(D_{n}^{(j)}\right)_{n \in \mathbb{N}}$ is supposed to be such that a well-defined density of states exists, namely a probability measure $\mathcal{N}_{j}$ such that

$$
\lim _{n \rightarrow \infty} \mathbf{E} \frac{1}{n} \operatorname{Tr}_{n}\left(f\left(D_{n}^{(j)}\right)\right)=\int \mathcal{N}_{j}(d x) f(x), \quad f \in C_{0}(\mathbb{R}) .
$$

The asymptotic behavior (in $n$ ) of the families $\left(X_{n}^{(i)}\right)_{i \in I}$ and $\left(D_{n}^{(j)}\right)_{j \in J}$ now defines a state $\varphi$ on the algebra $\mathcal{A}=\mathbb{C}\left[\left\{x^{(i)}\right\}_{i \in I},\left\{d^{(j)}\right\}_{j \in J}\right]$ of non-commutative polynomials by

$$
\begin{aligned}
\varphi\left(Q\left(\left\{x^{(i)}\right\}_{i \in I},\left\{d^{(j)}\right\}_{j \in J}\right)\right) \\
=\lim _{n \rightarrow \infty} \mathbf{E} \frac{1}{n} \operatorname{Tr}_{n}\left(Q\left(\left\{X_{n}^{(i)}\right\}_{i \in I},\left\{D_{n}^{(j)}\right\}_{j \in J}\right)\right), \quad Q \in \mathcal{A} .
\end{aligned}
$$

Theorem 2. The commutative subalgebras $\mathbb{C}\left[x^{(i)}\right]$ and $\mathbb{C}\left[d^{(j)}\right]$ are free in $(\mathcal{A}, \varphi)$ for all $i \in I$ and $j \in J$. Moreover, the marginals of $\varphi$ on $\mathbb{C}\left[x^{(i)}\right]$ and $\mathbb{C}\left[d^{(j)}\right]$ are respectively the semicircle law and the measure $\mathcal{N}_{j}$.

The first statement means, in particular, that all mixed moments of $\varphi$ can be calculated from the moments of the semicircle law and the densities $\mathcal{N}_{j}$ by use of free combinatorics discussed in detail in [Spe, HP]. In view of the above arguments, the proof of this theorem is a straightforward generalization of the one given in [Spe].

Acknowledgment: We would like to thank J. Bellissard, for bringing the flipmatrix models to our attention and explaining their link with the Anderson model, and M. Disertori, for discussions related to this work.

\section{References}

[Bel] J. Bellissard, Random matrix theory and the Anderson model, J. Stat. Phys. 116 (2004) 739-754 .

[BMR] J. Bellissard, J. Magnen, and V. Rivasseau, Supersymmetric analysis of a simplified two dimensional Anderson model at small disorder, Markov Processes and Related Fields 9 (2003) 261-278 .

[HP] F. Hiai, D. Petz, The semicircle law, free random variables and entropy, Amer. Math. Soc., Mathematical Surveys and Monographs 77 (2000).

[Poi] G. Poirot, Mean Green's function of the Anderson model at weak disorder with an infrared cutoff, Ann. Inst. Henri Poincaré 70 (1999) 101-146.

[Spe] R. Speicher, Lecture on free probability theory; in German, 1997/98, available at http://www. mast. queensu. ca/ speicher.

[Voi] D. Voiculescu, Limit laws for random matrices and free products, Invent. Math. 104 (1991) 201-220 .

[VDN] D. Voiculescu, K. Dykema, A. Nica, A noncommutative probability approach to free products with applications to random matrices, operator algebras and harmonic analysis on free groups, CRM Monograph Series. 1. Amer. Math. Soc., Providence, RI (1992).

[Wig] E. P. Wigner, Characteristic vectors of bordered matrices with infinite dimensions, Ann. of Math. 62 (1955) 548-564. 
Institut Für Theoretische Physik, ETH, Zürich, Switzerland

E-mail address: jschenker@itp.phys.ethz.ch

Mathematisches Institut, Universität Erlangen, Germany

E-mail address: schuba@mi.uni-erlangen.de 